\title{
The role of dietary history in the effects of novelty on taste aversions
}

\author{
DONNA M. ZAHORIK \\ Cornell University, Ithaca, New York 1485 s
}

\begin{abstract}
The novelty of taste stimuli is an important determinant of rats' taste preferences and taste aversions, but the effects of novelty may be overestimated in studies of laboratory-reared rats with very limited taste experience. The tendency to associate illness with novel rather than familiar flavors was compared in rats raised on standard laboratory diets and rats raised on varied diets including a number of distinctively flavored foods and liquids. The results suggest that the large effects of novelty are not simply artifacts produced by laboratory rats' limited taste experience, because both groups showed a strong tendency to associate illness with a novel taste rather than a familiar taste.
\end{abstract}

The familiarity of taste stimuli is an important parameter in rats' taste preferences, with both wild and albino rats showing general neophobia-a preference for familiar tastes (Barnett, 1963; Rozin, 1968). The strength of learned aversions to tastes which have been paired with illness also depends on the familiarity of the taste stimulus, with illness more readily associated with novel tastes than familiar tastes (Kalat \& Rozin, 1973; Maier, Zahorik, \& Albin, 1971; Revusky \& Bedarf, 1967). Although the familiarity-novelty variable clearly controls rats' selections of food and drink in all these studies, there is something unique about the context in which novel flavors are presented in typical taste experiments: laboratory-reared rats, including the rats used in taste aversion studies, have extremely limited experience with flavors of food and water prior to the experiment: Mother's milk, lab chow, and tap water may provide the total history of dietary taste experience for the laboratory animal. Certainly, a novel flavor which is not only new but also a member of an extremely small set of tastes experienced in a lifetime is different from a novel flavor which is only the most recent addition to a set of dozens or hundreds of flavors sampled over a period of time. In the context of very limited taste experience, the presentation of a novel flavor may be a far more powerful and aversive stimulus event than the presentation of the same novel taste to a rat with a more varied dietary history.

The idea that experience with novel tastes can alter the effects of taste familiarity is supported by at least two studies which demonstrate a reduction in neophobia toward a particular novel taste following preexposure to a different novel taste without any aversive consequences. Siegel (1974, Experiment 3) tested rats' consumption of either coffee or vinegar-flavored water in a one-bottle test following preexposure to the same taste (coffee-coffee or vinegar-vinegar), preexposure to the other taste (coffee-vinegar or vinegar-coffee), or preexposure to tap water. As expected, the rats preexposed to the same flavor on which they were tested drank more of that flavor than the rats preexposed to tap water; but the rats preexposed to a different novel flavor also drank more of the test flavor than the rats which had been preexposed to tap water. Experience with illness normally increases subsequent neophobia (Rozin, 1968), but Best (1975, Experiment 4a) found that this effect was reduced when rats were given "safe" exposures to a novel taste before they became ill; as in the Siegel experiment, preexposure to a novel taste reduced subsequent neophobia even when the preexposed flavor was different from the flavor later used in the test situation.

Whether these results are explained as examples of the generalization of "learned safety" following experience with a safe novel taste or as cases of habituation of the neophobia response, they suggest that the importance of the novelty-familiarity variable in rats' food preferences may be grossly overestimated in studies of laboratory-reared animals with severely limited taste experience. Because neophobia and taste aversion learning have often been viewed as complementary features of a highly evolved feeding system adapted to keeping wild rodents out of dietary trouble in their natural habitats, the suggestion that novelty effects may be artifacts produced by laboratory conditions which do not exist in the wild is disturbing and bears further study.

In the present experiment, the tendency to associate illness with novel (rather than familiar) tastes was compared in rats with very different dietary histories. Two groups of rats (Group $V_{F P}$, raised on varied diets, and Group $\mathrm{C}_{\mathrm{FP}}$, raised on tap water and lab chow) were presented with a familiar flavor (vanilla) and a novel flavor (saccharin) just before they were poisoned with lithium chloride. If experience with safe novel flavors attenuates the tendency to associate illness with novel rather than familiar flavors, we would expect Group $C_{F P}$ to show a stronger preference for the familiar flavor after poisoning than Group $V_{F P}$. To provide an accurate assessment of the effect of familiarity on associations between tastes and illness, two additional control groups 
Table 1

Experimental Design

\begin{tabular}{llllcl}
\hline & \multicolumn{4}{c}{ Experience with } \\
Group & Diet & Van & Sac & Poison & Test Flavors \\
\hline$V_{\text {FP }}$ & varied & Yes & No & Yes & van vs. sac \\
C FP $_{\text {FP }}$ & standard & Yes & No & Yes & van vs. sac \\
$C_{\text {NP }}$ & standard & No & No & Yes & van vs. sac \\
$C_{N}$ & standard & No & No & No & van vs. sac \\
\hline
\end{tabular}

raised on tap water and lab chow were employed: Group $C_{N P}$ was poisoned after drinking both vanilla and saccharin solutions, but both flavors were novel for group $\mathrm{C}_{\mathrm{NP}}$; Group $\mathrm{C}_{\mathrm{N}}$ served as a control for the effects of lithium chloride poisoning on taste preferences, receiving no pairing of tastes with illness before being tested on the two novel flavors.

\section{METHOD}

The subjects were 77 albino rats from eight litters bred from CD strain parents (Charles River Labs) and reared in our labs. Each litter was assigned at birth to one of two rearing conditions. The five litters assigned to Control Groups $C_{F P}, C_{N P}$, and $\mathrm{C}_{\mathrm{N}}$ were reared according to standard laboratory procedures: During gestation and after the birth of the litters, each mother rat was housed in a large, solid floor cage and supplied with tap water and lab chow ad lib. When the pups were 35 days old, they were moved to individual wire mesh cages, where they continued to receive tap water and lab chow exclusively until their 65 th day.

The three litters assigned to the varied diet condition (Group $V_{F P}$ ) received identical treatment with regard to caging and time of weaning, but they were provided with various foods and liquids throughout the course of development. The substances provided included B-complex test diet, chocolate milk, peanut butter cookies, Noyes pellets, apple slices, carrot juice, celery, cabbage juice, $.5 \% \mathrm{NaCl}$ solution, $.3 \%$ acetic acid solution, oatmeal, and milk; each litter received at least nine of these distinctively flavored substances during the first 64 days of life. The schedules on which these flavors were presented were not truly random because certain restrictions were necessary to insure the continuing good health of the subjects; nevertheless, the schedules were varied in the sense that the new foods and liquids were presented for various lengths of time, in various combinations, and some of the flavors were presented on several occasions. Because there is some evidence that adult rats play a role in controlling pups' food choices (e.g., Galef \& Clark, 1971), the presentation of novel flavors began while the pups were still receiving all their nourishment from milk, so that the mothers became familiar with some of the flavors before the pups were old enough to sample them. Both mothers and pups actually consumed all the foods and liquids which were presented, although some were obviously more palatable than others.

On the 65 th day, the litters assigned to Groups $V_{F P}$ and $C_{F P}$ were familiarized with a novel vanilla-flavored solution. The solution ( $50 \mathrm{ml}$ McCormick's vanilla extract/liter of tap water) was dispensed from 100-ml graduated cylinders and was the only source of fluid for the rats groups $V_{F P}$ and $C_{F P}$ for $24 \mathrm{~h}$. At the end of the familiarization period, bottles of tap water were returned to the cages, and no subject received a flavor other than tap water and lab chow until Day 68.

All subjects were deprived of water for $24 \mathrm{~h}$ prior to the experimental manipulations on Day 68. At the end of the deprivation period, each rat in Groups $\mathrm{V}_{\mathrm{FP}}, \mathrm{C}_{\mathrm{FP}}$, and $\mathrm{C}_{\mathrm{NP}}$ was given 5 min of access to each of two distinctively flavored solutions: vanilla solution $(50 \mathrm{ml}$ McCormick's vanilla extract/liter tap water) and saccharin solution $(2 \mathrm{~g}$ sodium saccharin/liter tap water). The flavors were presented sequentially and the order of presentation was counterbalanced by litters, so that approximately half of the animals in each group received vanilla followed by saccharin and the others received saccharin followed by vanilla. Rats which failed to drink at least $1 \mathrm{ml}$ of each flavor were dropped from the experiment, but since both flavors are highly palatable and the subjects were water deprived, most of the rats in all three groups drank both flavors readily. After presentation of both solutions, each rat was given an injection of $.3 \mathrm{M}$ lithium chloride (1\% body weight) and the water bottle was removed from the cage for $2 \mathrm{~h}$.

After $2 \mathrm{~h}$, two graduated cylinders fitted with drinking tubes were placed on each cage: The cylinder containing saccharin was always placed on the right and the one containing vanilla on the left, so that position and flavor were intentionally confounded. Intake of both flavors was measured after 2,8 , and $24 \mathrm{~h}$. Group $C_{N}$ was given the same two-bottle preference test between saccharin and vanilla solutions, but the animals in that group did not receive any pairing of the flavors with poisoning before the preference test.

Thus, all the subjects were given the same preference test on the 68th day after birth, but the groups differed with regard to their experience with novel flavors during development $(\mathrm{V}=$ varied diet, $C=$ standard diet), whether or not they were familiar with vanilla $(F=$ familiar, $N=$ novel) and whether or not they had been poisoned after drinking vanilla and saccharin $(P=$ poisoned). The treatments for all four groups are summarized in Table 1.

\section{RESULTS}

The results of the preference test are summarized in Table 2. The mean absolute intake of vanilla for each group is presented, as well as the mean preference score-the ratio of vanilla consumed to total fluic consumed during the test. The ordering of the fous groups with regard to both measures did not change oves time during the preference test, so only the data collec ted after $24 \mathrm{~h}$ are presented.

The data suggest that familiar flavors are less readily associated with poisoning than novel flavors, whether or not the subject has extensive experience with safe nove. flavors. Both groups which were familiar with vanille (Groups $V_{F P}$ and $C_{F P}$ ) drank large amounts of that fla vor during the test and showed large preferences for vanilla over saccharin. In contrast, the two groups which had not been exposed to vanilla prior to the test day (Groups $\mathrm{C}_{\mathrm{NP}}$ and $\mathrm{C}_{\mathrm{N}}$ ) showed strong preferences for saccharin over vanilla in the same test. There is some evidence that the animals raised on varied diet: (Group $\mathrm{V}_{\mathrm{FP}}$ ) drank less of the familiar solution than the rats which had been raised on tap water and lab chow $\left(C_{F P}\right)$, but the preference scores for those two groups were not significantly different.

These conclusions are supported by the results of two one-way analyses of variance performed on the un weighted means for the two sets of data. The mair effect of the four treatments on the amount of vanills consumed by the treatment groups was significan $[F(3,73)=50.77, p<.001]$, with the groups which were familiar with vanilla prior to poisoning $\left(\mathrm{V}_{\mathrm{FP}}, \mathrm{C}_{\mathrm{FP}}\right.$ ) drinking large amounts of vanilla in the preference tesi and the groups which were not familiar with vanilla 
Table 2

Mean Amounts and Proportions of Vanilla Solution Consumed by the Four Treatment Groups During the 24-H Preference Test

\begin{tabular}{|c|c|c|c|c|}
\hline Group & $\begin{array}{c}\text { Number of } \\
\text { Subjects }\end{array}$ & $\begin{array}{c}\text { Mean Amount } \\
\text { of Vanilla } \\
\text { Consumed } \\
(\mathrm{ml})\end{array}$ & $\begin{array}{c}\text { Mean } \\
\text { Proportion } \\
\text { Vanilla/Total } \\
\text { Fluid Consumed }\end{array}$ & $\begin{array}{c}\% \text { Extreme } \\
\text { Scorers in } \\
\text { Group }\end{array}$ \\
\hline $\mathrm{V}_{\text {FP }}$ & 24 & 49.8 & .85 & 92 \\
\hline $\mathrm{C}_{\mathrm{FP}}$ & 19 & 70.3 & .92 & 79 \\
\hline $\mathrm{C}_{\mathrm{NP}}$ & 20 & 3.9 & .06 & 95 \\
\hline $\mathrm{C}_{\mathrm{N}}$ & 14 & 20.9 & .22 & 21 \\
\hline
\end{tabular}

$\left(\mathrm{C}_{\mathrm{NP}}, \mathrm{C}_{\mathrm{N}}\right)$ drinking much smaller amounts of that flavor. Post hoc comparisons revealed that Group $V_{F P}$ drank significantly more vanilla solution than either Group $\mathrm{C}_{\mathrm{N}}$ or Group $\mathrm{C}_{\mathrm{N}} \quad(\mathrm{p}<.01$ for both comparisons); Group $\mathrm{C}_{\mathrm{FP}}$ also drank more vanilla solution than the other control groups $(\mathrm{p}<.01$ for both comparisons). Although familiarity with vanilla obviously accounted for much of the variance between groups, previous experience with safe novel flavors also contributed to the significant effect of treatment groups: The subjects raised on varied diets (Group $V_{F P}$ ) drank less vanilla solution than the subjects which were also familiar with vanilla but raised on standard lab diets (Group $\mathrm{C}_{\mathrm{FP}}$ ). The comparison between these groups was significant $(\mathrm{p}<.01)$.

The proportion of vanilla solution consumed/total fluid consumed also showed a strong effect of treatment groups $[\mathrm{F}(3,73)=63.37, \mathrm{p}<.001]$; that proportion was far larger for the groups familiar with vanilla $\left(V_{F} P\right.$ and $\left.C_{F P}\right)$ than for the groups which had not sampled vanilla prior to the test day $\left(\mathrm{C}_{\mathrm{NP}}\right.$ and $\left.\mathrm{C}_{\mathrm{N}}\right)$. All four comparisons between groups familiar with vanilla and groups unfamiliar with vanilla were significant $(p<.01)$. Even though the varied diet group consumed a smaller amount of vanilla solution than Group $\mathrm{C}_{\mathrm{FP}}$, the comparison between the preference scores for those two groups was not significant; so there was no evidence that rats raised on varied diets show a smaller preference for the familiar vanilla solution than rats raised on limited diets.

One of the most intriguing results of this experiment involves Group $C_{N}$, which was run as a check on the relative palatability of saccharin and vanilla in the absence of all experimental manipulations employed with the other groups. Although experience with illness has been shown to increase neophobia, there was no a priori reason to suspect that this control group would show different preferences than Group $\mathrm{C}_{\mathrm{N}}$, since both test flavors were novel for both groups. We might expect Group $\mathrm{C}_{\mathrm{NP}}$ to drink less of both flavors than Group $\mathrm{C}_{\mathrm{N}}$, but we would expect the proportion of vanilla consumed by both groups to be equal and to reflect the relative palatability of vanilla and saccharin solutions. Although Group $C_{N}$ did drink more vanilla $(M=20.9 \mathrm{ml})$ than Group $C_{N P}(M=3.9 \mathrm{ml})$ and a larger proportion of vanilla $\left(M=.22\right.$ vs. $M=.06$ for Group $\left.C_{N P}\right)$, neither difference was significant. Nevertheless, inspection of the raw data revealed one very striking difference between Group $\mathrm{C}_{\mathrm{N}}$ and all three poisoned groups, a difference that is not apparent when one considers only the means for the different treatments. Most of the animals in Group $\mathrm{C}_{\mathrm{N}}$ drank about $22 \%$ vanilla, as reflected in the mean preference score for that group, but virtually all of the poisoned animals drank close to $100 \%$ of one flavor or the other; the mean preference scores for the poisoned groups are not all $0 \%$ or $100 \%$, but deviations from those extremes are due primarily to a few animals in each group showing a reversal of preference rather than to any consistent sampling of both tastes. Counting all the subjects which drank $90 \%$ or more of one flavor as "extreme" scorers, Table 2 shows that almost all the animals in Groups $\mathrm{V}_{\mathrm{FP}}, \mathrm{C}_{\mathrm{FP}}$, and $\mathrm{C}_{\mathrm{NP}}$ are extreme scorers, while only $21 \%$ of the rats in Group $\mathrm{C}_{\mathrm{N}}$ fit that description. The distribution of extreme scorers among the treatment groups is not random, but depends on the treatment $\left[\chi^{2}(3)=25.46, p<.001\right]$. Experience with illness seems to promote a certain exclusivity in situations where a rat is allowed a choice of flavors: Factors such as the relative familiarity of the tastes (Groups $V_{F P}$ and $C_{F P}$ ) or the relative palatability of flavors (Group $\mathrm{C}_{\mathrm{NP}}$ ) determined which flavor was chosen, but experience with illness determined whether or not a clear choice was made.

\section{DISCUSSION}

Many experiments have demonstrated the importance of taste novelty in controlling rats' taste preferences. Familiar flavors are generally preferred to novel flavors, and familiar flavors are also less readily associated with gastrointestinal illness than are novel flavors. Yet the effects of novelty have usually been studied in the context of the laboratory rat's very limited taste experience, and there are both theoretical and empirical reasons to believe that this context may magnify the importance of novelty in food selection. The results of the present experiment suggest that the frequently observed tendency to associate illness with novel rather than familiar tastes is not produced, or even greatly enhanced, by limited taste experience. Rats raised on varied diets from birth did not perform very differently than rats raised on standard laboratory diets in their readiness to associate illness with a novel rather than a familiar flavor in a standard taste aversion paradigm.

It is not clear why exposure to many safe novel flavors should have little effect on later responses to novel flavors when preexposure to a single safe novel taste has been shown to reduce subsequent neophobia (Best, 1975; Siegel, 1974). One possible explanation is that learned safety produced by exposure to a safe novel flavor does not generalize equally to all other novel flavors, but generalizes according to some gradient of stimulus similarity. If that is the case, the particular flavors chosen for preexposure and testing may account for the very small effects of preexposure to various tastes on later responses 
to vanilla in this experiment. The possible importance of such generalization gradients in taste preferences is illustrated by recent studies which suggest that the increased "nonspecific" neophobia observed following experience with illness is actually dependent on the particular flavor paired with poison and its relation to the flavor used in the neophobia test (Domjan, 1975).

\section{REFERENCES}

BARnett, S. A. The rat: A study in behavior. Chicago: Aldine, 1963.

BESt, M. R. Conditioned and latent inhibition in tasteaversion learning: Clarifying the role of learned safety. Journal of Experimental Psychology: Animal Behavior Processes. 1975. 104, 97-113.

Domjan. M. Poison-induced neophobia in rats: Role of stimulus generalization of conditioned taste aversions. Animal Learning \& Behavior, 1975, 3, 205-211.

Galef, B. G., \& Clark, M. M. Social factors in the poison avoidance and feeding behavior of wild and domesticated rat pups. Journal of Comparative and Physiological Psychology. 1971. 75. 341-357.
Kalat, J. W., \& Rozin, P. "Learned safety" as a mechanism in long-delay taste aversion learning in rats. Journal of Comparative and Physiological Psychology. 1973. 83. 198-207.

Maier, S. F., Zahorik, D. M.. \& Albin, R. W. Relative novelty of solid and liquid diet during thiamine deficiency determines development of thiamine-specific hunger. Journal of Comparative and Physiological Psychology. 1971, 74. 254-262.

Revusky, S. H., \& Bedarf, E. W. Association of illness with prior ingestion of novel foods. Science, 1967, 155, 219-220.

Rozin, P. Specific aversions and neophobia resulting from vitamin deficiency or poisoning in half-wild and domestic rats. Journal of Comparative and Physiological Psychology. 1968, 66, 82-88.

SiEgel. S. Flavor preexposure and "learned safety." Journal of Comparative and Physiological Psychology, 1974, 87. 1073-1082.

(Received for publication June 21, 1976.) 